\title{
Need probability affects retention: A direct demonstration
}

\author{
RICHARD B. ANDERSON, RYAN D. TWENEY, MARK RIVARDO, and SEAN DUNCAN \\ Bowling Green State University, Bowling Green, Ohio
}

\begin{abstract}
Recent memory theory has emphasized the concept of need probability - that is, the probability that a given piece of learned information will be tested at some point in the future. It has been proposed that, in real-world situations, need probability declines over time and that the memory-loss rate is calibrated to match the progressive reduction in need probability (J. R. Anderson \& Schooler, 1991). The present experiments were designed to examine the influence of the slope of the need-probability curve on the slope of the retention curve. On each of several trials, subjects memorized a list of digits, then retained the digits in memory for $1,2,4,8$, or 16 sec. Some trials ended with a recall test; other trials ended with the message, "no test." In Experiment 1, the likelihood of encountering a memory test (i.e., the need probability) was made to either increase or decrease as the retention interval increased; in Experiment 2, need probability either was flat (invariant across retention intervals) or decreased as the retention interval increased. The results indicated that the shape of the need-probability curve influenced the slope of the retention curve (Experiment 1) and that the effect became larger as the experimental session progressed (Experiment 2). The findings support the notion that memory adapts to need probabilities and that the rate of forgetting is influenced by the slope of the need-probability curve. In addition, all of the forgetting curves approximated a power function, suggesting that need probability influences the slope but not the form of forgetting.
\end{abstract}

The power function has been regarded as an appropriate mathematical description of human performance both in sensation (Stevens, 1971) and in skill acquisition (e.g., J. R. Anderson, 1982; Logan, 1988; Newell \& Rosenbloom, 1981). In addition, recent findings show that the power function describes how memories decline over time (J. R. Anderson \& Schooler, 1991; Rubin, 1982; Wixted \& Ebbesen, 1991). Although several theories offer a process-level account of power-law skill acquisition (e.g., J. R. Anderson, 1982; Logan, 1988), there have been few attempts to explain power-law forgetting. One exception is $\mathbf{J}$. R. Anderson's adaptive theory of memory, in which the retention function can be understood as adaptive to the informational environment (J. R. Anderson, 1990; J. R. Anderson \& Milson, 1989).

In evaluating the adaptation hypothesis, J. R. Anderson and Schooler (1991) conducted several studies of real-world informational environments. They found that need probability does decline as a power function of the retention interval, though they conceptualized retention interval in a novel way. In one study, for example, J. R. Anderson and Schooler calculated a particular word's occurrence probability - that is, the probability that it would appear in the current issue of the New York Times-as a function of its repetition lag (i.e., the number of days since its last occurrence). Figure 1 shows the results averaged

The authors thank John Wixted, Donald Bamber, and Geoffrey Lof tus for their comments on earlier drafts of the manuscript. Correspondence should be addressed to R. B. Anderson, Department of Psychology, Bowling Green State University, Bowling Green, OH 43403 (e-mail: randers@trapper.bgsu.edu). over a set of words. The data were fit well by the power function, $P=A t^{-B}$, where $P$ is the performance measure, $t$ is time, and $A$ and $B$ are parameters. For the New York Times data, $A=0.15$, and $B=0.73$, and the power function accounted for $99 \%$ of the variance. J. R. Anderson and Schooler argued that the similarity between the lexical occurrence-probability function and the classic memory retention function is no mere coincidence but reflects adaptation of the memory system to its informational environment. The argument holds that each occurrence of a word creates a need to retrieve information relevant to that word. Thus, the occurrence probability is equivalent to the need probability. A well-adapted memory system with limited resources will retain information that has high need probability and will forget that which has low need probability. Consequently, if need probability for a given piece of information within a given environment declines as a power function of time, then optimal memory performance will be characterized by a retention curve that approximates a power function.

J. R. Anderson and Milson (1989) cited the spacing effect (Glenberg, 1976; Keppel, 1964) as an example of how need probability influences the slope of the retention curve in a standard laboratory task. In one version of the task, subjects learned lists of paired associates, then recalled them either immediately or after a 1- or 8-day retention interval (Keppel, 1964). The results showed that wide spacing of study trials yielded a slower rate of forgetting than did close spacing. More specifically, when retention was tested immediately after learning, recall was higher for items studied under close-spacing conditions than under wide-spacing conditions. However, the oppo- 


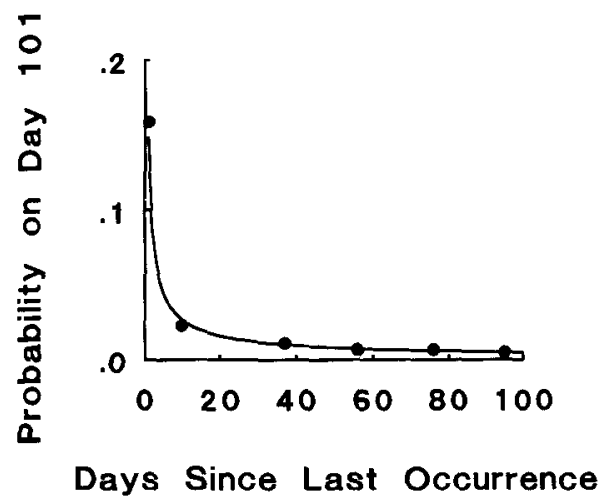

Figure 1. Probability of a word's appearance in the New York Times on Day 101 (i.e., the current issue) as a function of the amount of time since its last appearance. Solid line shows bestfitting power curve. Adapted from "Reflections of the Environment in Memory," by J. R. Anderson \& L. J. Schooler, 1991, Psychological Science, 2, p. 403. Copyright 1991 by American Psychological Society. Adapted with permission.

site effect occurred after a 1- or 2-day retention interval: Recall was higher for items studied under wide-spacing conditions than under close-spacing conditions. Keppel's results were replicated by a portion of Glenberg's (1976, Experiment 1) data, which showed that the forgetting rate generally decreased as the spacing interval increased from 4 to $40 \mathrm{sec}$. As an aside, the data for more closely spaced study trials in Glenberg's experiment yielded an anomalous finding: One-second spacing between study trials yielded a forgetting rate that was slower than that produced by either 0 - or 4-sec spacing. Still, the overall pattern matched Keppel's.

Why should forgetting be generally slower following wide spacing than following close spacing? J. R. Anderson and Milson (1987) offered the following explanation. During study, each stimulus repetition creates a need to retrieve information relevant to the stimulus. As a result, subjects learn that when repetitions are closely spaced, need probability is maximal during time periods immediately following the most recent repetition. However, when spacing is wide, subjects learn that the need probability is maximal after long time intervals, corresponding to the wide spacing of the study trials. The need-probability curves learned during acquisition are then carried over to the retention period, causing the wide-spacing condition to yield retention performance that starts off lower but that ends up higher than does retention in the close-spacing condition. J. R. Anderson and Milson (1987) used the term history factor as a general descriptor for such carryover effects.

There are alternatives to need probability as an explanation for spacing effects. Wide spacing of repetitions may provide an opportunity for subjects to receive feedback about the effectiveness of their encoding strategies: Given sufficient time between study trials, subjects may forget earlier repetitions and may thereby be prompted to try new strategies that complement earlier ones (Melton, 1970) or that improve upon earlier ones (Keppel, 1967).
Thus, while the learning of need probabilities seems a plausible explanation for the spacing effect, the phenomenon is also consistent with other theoretical accounts.

\section{NEED PROBABILITY AND TEST PROBABILITY: RATIONALE FOR THE PRESENT EXPERIMENTS}

Need probability may affect the retention function by means other than carryover effects. J. R. Anderson and Milson (1989) suggested that the context factor-that is, contextual cues within the retrieval environment-may influence people's estimate of need probability. In the present paper, we extend J. R. Anderson and Milson's point by arguing that test probability, an unexamined context factor present in every laboratory study of memory, may substantially moderate the effect of the retention interval on memory retrieval.

Certainly, test probability is closely related to need probability; in a recall or recognition test, the objective likelihood of needing to retrieve a particular piece of information is virtually identical to the likelihood of being tested on that information. In a Brown-Peterson type experiment (Brown, 1958; Peterson \& Peterson, 1959), each trial consists of a to-be-remembered set of targets, followed by a list of distractors that are processed (e.g., named) but not memorized, followed by a test of recall for the targets. Trials usually differ in the lengths of their retention intervals (RIs). Because there is an equal number of trials - including an equal number of tests-at each RI, the test-probability function is flat. That is, the probability of being tested is equal, and usually 1.0 , for all RIs.

There is already evidence that test probability can influence recall. Muter (1980) found that subjects exhibited higher or lower levels of recall, depending on whether the test probability was high or low, respectively. It is possible, however, that test probability affects recall in more subtle ways. Assuming that test probability affects need probability, and that need probability influences the retention function, manipulating the shape of the testprobability curve should affect the shape of the retention function. Such a finding would support the theory that memory performance reflects the need probabilities in the environment.

\section{EXPERIMENT 1}

On each trial, the subjects vocalized a list of three to-beremembered numbers, then vocalized the names of distractor numbers for a period of $1,2,4,8$, or $16 \mathrm{sec}$. Some trials ended with a test of recall for the initial three numbers. The experiment was structured so that the subjects were exposed either to an upward-sloping test-probability curve or to a downward-sloping test-probability curve. In the upward-sloping condition, the percentage of tested trials rose from $29.4 \%$ at the shortest RI to $100 \%$ at the longest RI (i.e., the ratios of test to no-test trials rose from $5: 12$ to $17: 0$ ). In the downward-sloping condition, the percent- 
age dropped from $100 \%$ at the shortest RI to $29.4 \%$ at the longest RI. It was predicted that the test-probability curves would influence the retention-probability curves in that the upward-sloping test-probability condition should yield a flatter retention function than should the downward sloping condition.

In addition, the subjects should respond to test probabilities only after having learned them through practice. Therefore, it was predicted that the retention slopes for the two test-probability conditions should differ more in later trials than in early trials.

\section{Method}

Subjects. There were 20 subjects in the experiment. Their participation partially fulfilled the requirements for an undergraduate course in cognitive psychology. All subjects were naive to the hypotheses under study.

Apparatus and Equipment. Stimulus presentation and data collection were performed using a microcomputer, VGA monitor, and Micro Experimental Laboratory software (Schneider, 1988).

Design and Procedure. Each subject performed a total of 85 trials, with 17 trials in each of five blocks. Table 1 contains the frequency of test and no-test trials at each RI in each of the two testprobability conditions. Each trial contained the following sequence of events: a warning signal, a list of numbers to be memorized (targets), a random and nonrepeating sequence of two-digit numbers (distractors), and either a recall test for the to-be-memorized numbers or a message saying "no test." All stimuli appeared against a dark background in the center of the monitor. The warning signal was a dash that persisted for $1.5 \mathrm{sec}$. The target numbers were printed in red; the distractor numbers were printed in white. The subjects were instructed to pronounce all numbers aloud but to memorize only the targets. To encourage compliance with instructions to say the numbers aloud, the subjects were required to vocalize into a microphone that was attached to a tape recorder. The numbers, including targets and distractors, were presented at a rate of one per second with an interstimulus interval of $200 \mathrm{msec}$. The subjects had $30 \mathrm{sec}$ to recall the targets and to type them into the computer. They were instructed to type exactly three two-digit numbers and to guess if necessary. The intertrial interval was $1.5 \mathrm{sec}$.

Test-probability condition, upward or downward sloping, varied between subjects. RI and block varied within subjects. The session, for each subject, lasted approximately $30 \mathrm{~min}$.

\section{Results and Discussion}

The mean number of recalled targets was calculated at each of five RIs in the early blocks (1-3) and in the late blocks ( 4 and 5 ) for each of 20 subjects, yielding a total of 40 retention curves. A preliminary analysis was conducted on the aggregate curves, averaged across sub-

Table 1

Total Number of Trials per Subject in Experiment 1 for the Upward-Sloping and Downward-Sloping Test-Probability Conditions for Test-Present and Test-Absent Trials

\begin{tabular}{crrrrr}
\hline $\begin{array}{c}\text { Postretention } \\
\text { Test }\end{array}$ & \multicolumn{5}{c}{ Retention Interval (in seconds) } \\
\cline { 2 - 6 } & 1 & 2 & 4 & 8 & 16 \\
\hline Present & 5 & 8 & 11 & 14 & 17 \\
Absent & 12 & 9 & 6 & 3 & 0 \\
& & Upward & & \\
Present & 17 & 14 & 11 & 8 & 5 \\
Absent & 0 & 3 & 6 & 9 & 12 \\
\hline
\end{tabular}

jects, to assess the general fit of the data to power, logarithmic, exponential, and linear functions. Table 2 shows the error in each function's fit, as measured by root mean squared error (RMSE). In all cases, the power fit yielded the smallest RMSE. Consequently, a nonlinear regression algorithm was used to fit the data to power functions of the form, $P=A t^{-B}$, where $P$ is the mean number recalled, $t$ is the length of the RI, $A$ is alpha (i.e., the intercept, the value of $P$ when $t$ is 1.0 ), and $B$ is beta (i.e., the slope). The curve-fitting procedure yielded two curves per subject-one for the early blocks and one for the late blocks - for a total of 40 power curves.

Using the method described by Loftus and Masson (1994), 95\% confidence intervals were constructed to analyze the effects of test probability (upward vs. downward sloping) and block (early vs. late) on the mean beta. A similar analysis was conducted on alpha. Figure 2 shows the results, including plots of the aggregate curves, and the confidence intervals for alpha and beta.

Alpha was significantly higher in the downward test condition than in the upward test condition in the early blocks only (the least significant difference, LSD, was 0.171 ). The result was difficult to interpret because there were no clear predictions concerning alpha. However, the effects on the slope (beta) confirmed the major prediction: Beta was significantly higher in the downwardsloping test condition than in the upward-sloping test condition both in the early block and in the late block $(\mathrm{LSD}=0.079)$. Though the slope difference was expected to increase with practice, the effects on beta were approximately equal in the early and late blocks. Perhaps the subjects learned the test probabilities extremely quickly, thereby masking a potential block effect. Overall, the data supported the notion that the slope of the retention curve adapts to the shape of the need-probability curve.

In addition to confirming the major predictions, the present data replicated earlier findings, showing that retention declines as a power function of time (J. R. Anderson \& Schooler, 1991; Wixted \& Ebbesen, 1991). As noted by Loftus and Bamber (1990), Wixted and Ebbesen (1991), and others, the choice of measurement scale can, in principal, affect the mathematical form of an empirically derived forgetting function. It has been shown, however, that various indices of memory performance, including proportion recalled and savings in relearning, produce the same empirical function--that is, a power function (Wixted \& Ebbesen, 1991; but see R. B. Anderson \& Tweney, 1997, for a discussion of artifactual power curves). Thus, the present results converge with the general finding that forgetting obeys a power law.

\section{EXPERIMENT 2}

Most studies of retention have incorporated flat needprobability functions. That is, the studies have contained equal test probabilities for all RIs that were present in the study. Because this contrasts with the downward-sloping, real-world, need-probability functions observed by J. R. Anderson and Milson (1989), it is reasonable to ask 
Table 2

RMSE for the Early and Late Blocks in the Upward-Sloping and Downward-Sloping Test-Probability Conditions in Experiment 1

\begin{tabular}{lcccc}
\hline & \multicolumn{4}{c}{ Function } \\
\cline { 2 - 4 } & Power & Logarithmic & Exponential & Linear \\
\hline Upward & 0.27 & Early Blocks & & \\
Downward & 0.33 & 0.47 & 0.62 & 1.70 \\
& \multicolumn{5}{c}{ Late Blocks } \\
Upward & 0.33 & 0.49 & 0.60 & 2.43 \\
Downward & 0.34 & 0.58 & 0.71 & 1.62 \\
\hline
\end{tabular}

Note--Data were fit to the following equations, where $P$ is performance, $t$ is time, and $e$ is the base of natural logs. For the power function, $P=A t^{-B}$; for the logarithmic function, $P=A-B \log (t)$; for the exponential function, $P=A e^{-B t}$; for the linear function, $P=A-B t$.

whether the retention curves yielded by most memory experiments contain a bias that results from using ecologically invalid test probabilities. The present experiment examined the effect of flat versus downward-sloping need probabilities on the form of the retention function. The results were expected to replicate the findings of Experiment 1 , though the present need-probability manipulation was relatively weak (the difference between flat and downward sloping is less extreme than is the difference between upward sloping and downward sloping). Additionally, the present experiment addressed the question of whether flat test probabilities can bias the slope of the retention function.

\section{Method}

Thirty subjects participated in the experiment to partially fulfill the requirements for an introductory psychology course. All subjects were naive to the hypotheses under study. The method was similar to that of Experiment 1, except that the present experiment substituted a flat test-probability condition in place of the upward-sloping condition of Experiment 1 . The two test-probability conditions contained an equal number of trials. In the flat condition, however, the percentage of tested trials was $64.7 \%$ (a test:no-test ratio of $11: 6$ ) for all RIs. The two experiments were identical in all other respects.

\section{Results and Discussion}

Table 3 shows RMSE, for the power, logarithmic, exponential, and linear fits to the aggregate data. The power

Blocks 1-3
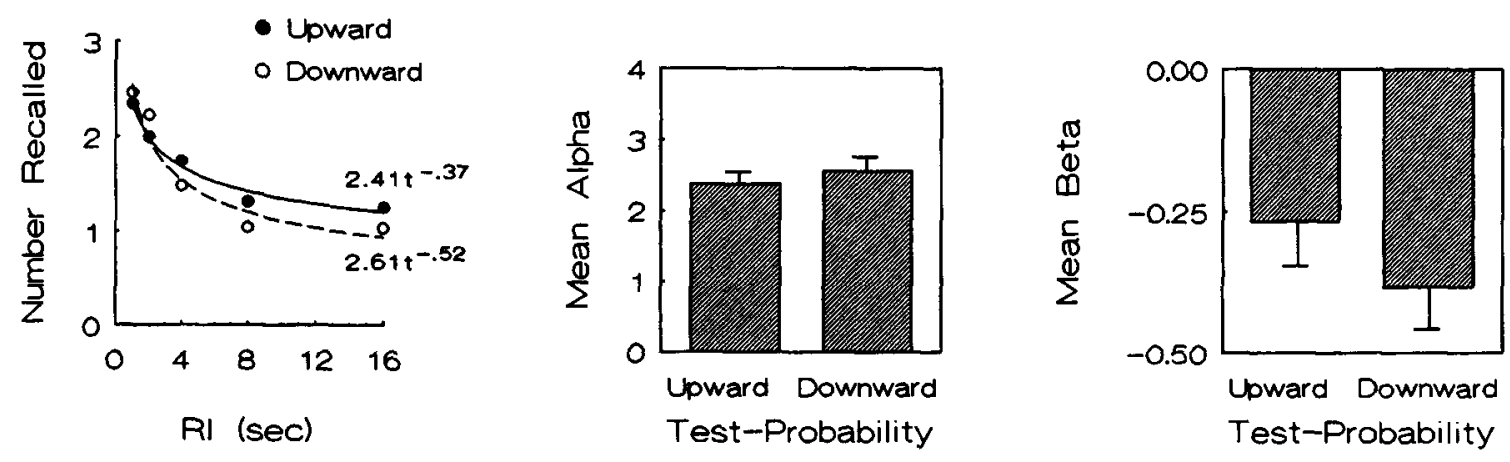

Blocks 4 \& 5

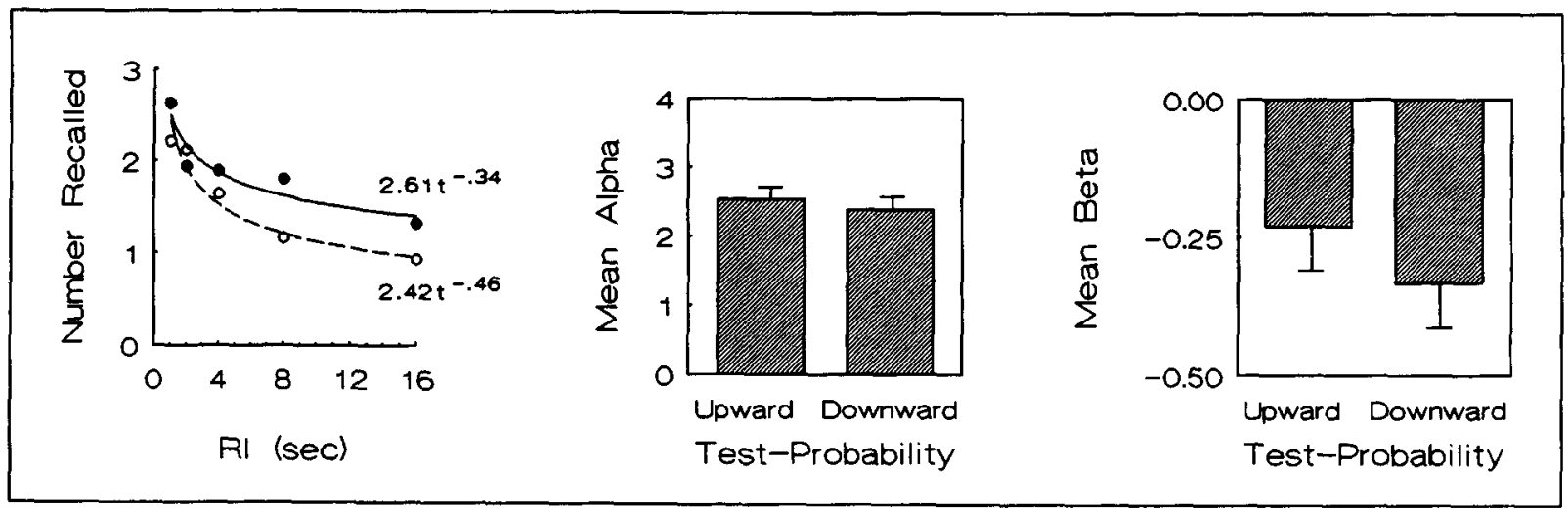

Figure 2. Experiment 1 data. Leftmost graphs show mean number recalled as a function of retention interval (RI) and test-probability condition, with best-fitting power functions. Algebraic expressions show the function parameters, where $t=$ time (i.e., retention interval). Middle and rightmost graphs show the effect of test-probability condition on the mean intercept (alpha) and the mean slope (beta). Error bars show $95 \%$ confidence intervals. 
function yielded the smallest error variance in all cases. Consequently, as in Experiment 1, each subject's curves (in the early and late blocks) were fit to power functions. Figure 3 shows the aggregate curves, as well as the confidence intervals for alpha and beta. There were no significant effects on alpha $(\mathrm{LSD}=0.131)$. However, there was a significant effect on beta in the late blocks only $(L S D=0.050)$. The results therefore indicate that forgetting adapts to need probabilities, as shown in Experiment 1 , and that the amount of adaptation tends to increase with practice.

The present findings generally replicated those of Experiment 1 but also showed that flat test probabilities, an almost universal feature of memory experiments, can affect the slope of the retention function. The block effect may indicate that the subjects gradually learned the test probabilities as the session progressed: Apparently, the weak manipulation of test probability in the present experiment (relative to that in Experiment 1) resulted in slower learning of the test probabilities. The results support the view that memory adapts to need probabilities in the environment and suggest that the standard method
Table 3

RMSE for the Early and Late Blocks in the Upward-Sloping and Downward-Sloping Test-Probability Conditions in Experiment 2

\begin{tabular}{lcccc}
\hline & \multicolumn{4}{c}{ Function } \\
\cline { 2 - 5 } & Power & Logarithmic & Exponential & Linear \\
\hline \multirow{4}{*}{ Early Blocks } \\
Upward & 0.29 & 0.59 & 0.61 & 2.09 \\
Downward & 0.30 & 0.62 & 0.62 & 2.12 \\
& \multicolumn{5}{c}{ Late Blocks } \\
Upward & 0.30 & 0.47 & 0.67 & 1.53 \\
Downward & 0.32 & 0.61 & 0.58 & 2.35 \\
\hline
\end{tabular}

Note - Data were fit to the following equations, where $P$ is performance, $t$ is time, and $e$ is the base of natural logs. For the power function, $P=A t^{-B}$; for the logarithmic function, $P=A-B \log (t)$; for the exponential function, $P=A e^{-B i}$; for the linear function, $P=A-B t$.

for measuring the form of forgetting may distort the slope of the function; flat need probabilities yield a flattened retention function. Also, as in Experiment 1, adaptation appeared to take place within the bounds of the power law.
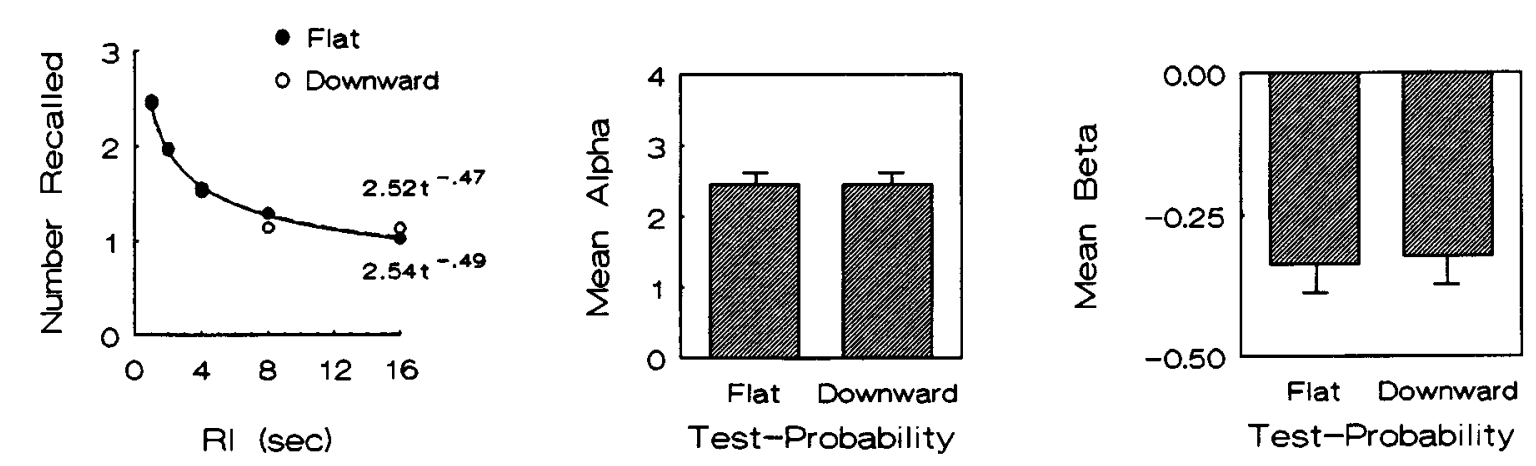

Blocks 4 \& 5

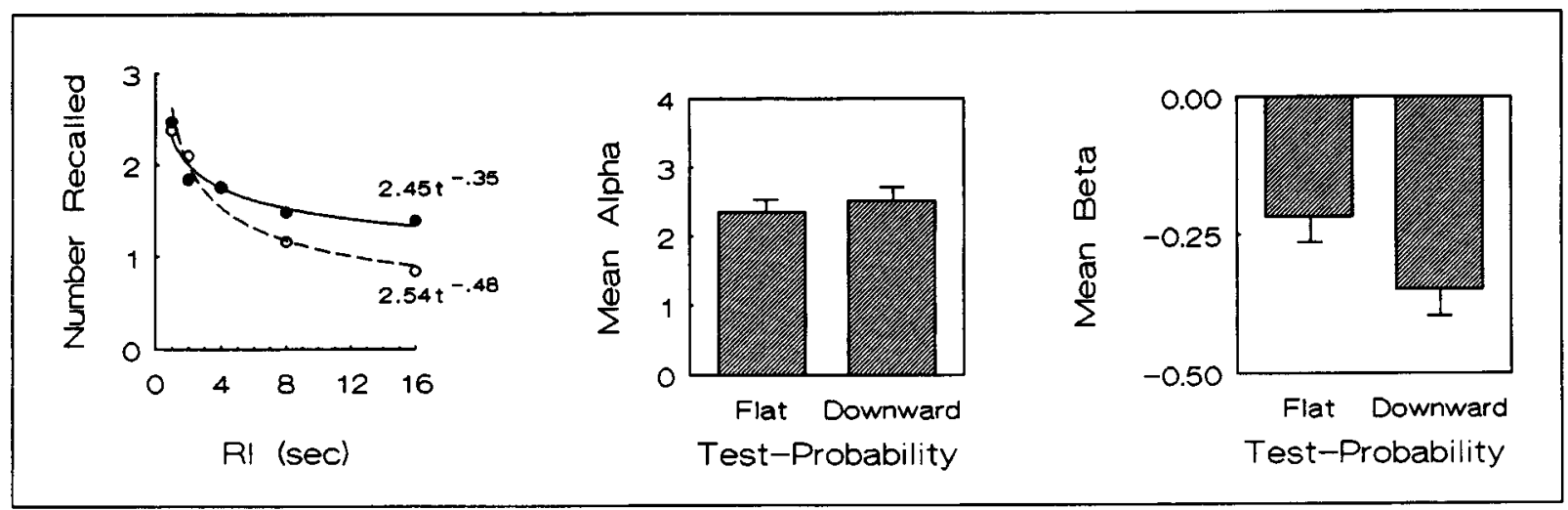

Figure 3. Experiment 2 data. Leftmost graphs show mean number recalled as a function of retention interval (RI) and test probability condition, with best-fitting power functions. Algebraic expressions show the function parameters, where $t=$ time (i.e., retention interval). Middle and rightmost graphs show the effect of test-probability condition on the mean intercept (alpha) and the mean slope (beta). Error bars show $95 \%$ confidence intervals. 


\section{GENERAL DISCUSSION AND CONCLUSIONS}

In the spirit of the adaptive approach to cognitive research, the present experiments have not emphasized processing mechanisms. Indeed, need-probability theory (J. R. Anderson, 1990) concerns the match between performance and the environment, and, therefore, it is compatible with a wide range of possible mechanisms. Still, the mechanisms are important. In the present experiment, the subjects' adaptive behavior may have involved strategic target processing either before the onset of the retention interval or during the RI. If the adaptation involved encoding prior to the onset of the RI, then, at the shortest RI, recall should have been much higher in the downwardsloping test condition than in the other condition (reflecting the very large difference in test probability). However, the absence of a clear need-probability effect at the shortest intervals (shown in Figures 1 and 2), together with the trend toward greater curve separation at longer RIs, suggests that adaptive processing occurred during the interval, not before. Apparently, the subjects were able to extend target encoding into the RI, perhaps through covert rehearsal, and were more likely to do so when there was a high need to retain the information over a long period of time. This interpretation is consistent with the data, despite the subjects' informal reports that they were unable to rehearse while naming the distractors.

In contrast to the foregoing argument, it is possible that adaptation involves the quality of encoding prior to the onset of the RI. Previous studies have found that the total encoding time (prior to RI onset) influences the intercept (alpha) but not the slope of the retention function (Wixted \& Ebbesen, 1991). However, need probability in the present experiments may have affected the quality rather than the quantity of encoding and therefore may have been capable of causing the slope differences in the data. If preretention encoding underlies the needprobability effect, then, arguably, the present results should have shown recall differences at every RI. Such was not the case. However, the absence of a test-probability effect at the shortest intervals may have been due to a ceiling effect: Retention may have remained high at short intervals, regardless of the test probability and regardless of the encoding quality. This interpretation is consistent with Muter's data showing little effect of test probability on immediate recall (Muter, 1980).

In the present experiments, the shape of the needprobability curve affected the slope of the retention curve, directly supporting J. R. Anderson's memory adaptation hypothesis. However, there was no apparent effect on the underlying form of the retention curve: The power function, in all cases, provided the best fit to the data. The results therefore support the notion that the power function is a fundamental characteristic of forgetting (J. R. Anderson \& Schooler, 1991) and that memory adaptation is constrained by the power law.

\section{REFERENCES}

Anderson, J. R. (1982). Acquisition of cognitive skill. Psychological Review, 89, 369-406.

ANDERSON, J. R. (1990). The adaptive character of thought. Hillsdale, $\mathrm{NJ}$ : Erlbaum.

ANDERSON, J. R., \& Milson, R. (1989). Human memory: An adaptive perspective. Psychological Review, 96, 703-719.

ANDERSON, J. R., \& SCHOOLER, L. J. (1991), Reflections of the environment in memory. Psychological Science, 2, 396-408.

ANDERSON, R. B., \& TWENEY, R. D. (1997). Artifactual power curves in forgetting. Memory \& Cognition, 25, 724-730.

BRown, J. A. (1958). Some tests of the decay theory of immediate memory. Quarterly Journal of Experimental Psychology, 10, 12-21.

GlenderG, A. M. (1976). Monotonic and nonmonotonic lag effects in paired-associate and recognition memory paradigms. Journal of Verbal Learning \& Verbal Behavior, 15, 1-16.

KEPPEL, G. (1964). Facilitation in short- and long-term retention of paired associates following distributed practice in learning. Journal of Verbal Learning \& Verbal Behavior, 3, 91-111.

KEPPEL, G. (1967). A reconsideration of the extinction-recovery theory. Journal of Verbal Learning \& Verbal Behavior, 6, 476-486.

LofTUs, G. R., \& BAMBER, D. (1990). Learning-forgetting independence, unidimensional memory models, and feature models: Comment on Bogartz (1990). Journal of Experimental Psychology: Learning, Memory, \& Cognition, 16, $916-926$.

LofTUs, G. R., \& Masson, M. E. J. (1994). Using confidence intervals in within-subject designs. Psychonomic Bulletin \& Review, 1, 476-490.

LogAN, G. D. (1988). Toward an instance theory of automatization Psychological Review, 95, 492-527.

MeLton, A. W. (1970). The situation with respect to the spacing of repetitions and memory. Journal of Verbal Learning \& Verbal Behavior, 9, 596-606.

MUter, P. (1980). Very rapid forgetting. Memory \& Cognition, 8 , 174-179.

Newell, A., \& Rosenbloom, P. S. (1981). Mechanisms of skill acquisition and the law of practice. In J. R. Anderson (Ed.), Cognitive skills and their acquisition (pp. 1-55). Hillsdale, NJ: Erlbaum.

Peterson, L. R., \& Peterson, M. J. (1959). Short-term retention of individual verbal items. Journal of Experimental Psychology, 58, 193-198.

RuBIN, D. C. (1982). On the retention function for autobiographical memory. Journal of Verbal Learning \& Verbal Behavior, 21, 21-38.

SCHNEIDER, W. (1988). Micro Experimental Laboratory: An integrated system for IBM PC compatibles. Behavior Research Methods, Instruments, \& Computers, 20, 206-217.

STEVENS, S. S. (1971). Neural events and the psychophysical law. Science, 170, 1043-1050.

WiXted, J. T., \& EbBesen, E. B. (1991). On the form of forgetting. Psychological Science, 2, 409-415.

(Manuscript received December 1, 1995; revision accepted for publication June 1, 1996.) 